\title{
$\Psi$-Stability of Nonlinear Volterra Integro-Differential Systems with Time Delay
}

\author{
Lianzhong Li, ${ }^{1,2}$ Maoan Han, ${ }^{1}$ Xin Xue, ${ }^{2}$ and Yuanyuan Liu ${ }^{1}$ \\ ${ }^{1}$ Department of Mathematics, Shanghai Normal University, Shanghai 200234, China \\ ${ }^{2}$ School of Mathematics and Statistics, Taishan University, Taian, Shandong 271021, China \\ Correspondence should be addressed to Lianzhong Li; 1lz3497@163.com
}

Received 2 March 2013; Revised 8 April 2013; Accepted 11 April 2013

Academic Editor: Marcia Federson

Copyright (C) 2013 Lianzhong Li et al. This is an open access article distributed under the Creative Commons Attribution License, which permits unrestricted use, distribution, and reproduction in any medium, provided the original work is properly cited.

We give some sufficient conditions for $\Psi$-uniform stability of the trivial solutions of a nonlinear differential system and of nonlinear Volterra integro-differential systems with time delay.

\section{Introduction}

Akinyele [1] introduced the notion of $\Psi$-stability of the degree $k$ with respect to a function $\Psi \in C\left(R_{+}-R_{+}\right)$, increasing and differentiable on $R$ and such that $\Psi(t) \geq 1$ for $t \geq 0$ and $\lim _{t \rightarrow \infty} \Psi(t)=b, b \in[1, \infty)$. Constantin [2] introduced the notions of degree of stability and degree of boundedness of solutions of an ordinary differential equation, with respect to a continuous positive and nondecreasing function $\Psi: R_{+} \rightarrow$ $R_{+}$; some criteria for these notions are proved there too.

Morchało [3] introduced the notions of $\Psi$-stability, $\Psi$ uniform stability, and $\Psi$-asymptotic stability of trivial solution of the nonlinear system $x^{\prime}=f(t, x)$. Several new and sufficient conditions for the mentioned types of stability are proved for the linear system $x^{\prime}=A(t) x$; in this paper $\Psi$ is a scalar continuous function. In $[4,5]$, Diamandescu gives some sufficient conditions for $\Psi$-asymptotic stability and $\Psi$-(uniform) stability of the nonlinear Volterra integrodifferential system $x^{\prime}=A(t) x+\int_{0}^{t} F(t, s, x(s)) d s$; in these papers $\Psi$ is a matrix function. Furthermore, in [6], sufficient conditions are given for the uniform Lipschitz stability of the system $x^{\prime}=f(t, x)+g(t, x)$.

In paper [7], for the nonlinear system

$$
y^{\prime}=f(t, y)+g(t, y)
$$

and the nonlinear Volterra integro-differential system

$$
z^{\prime}=f(t, z)+\int_{0}^{t} F(t, s, z(s)) d s,
$$

by using the knowledge of fundamental matrix and nonlinear variation of constants, we give some sufficient conditions for $\Psi$-(uniform) stability of trivial solution for the system. The purpose of this paper is to provide sufficient conditions for $\Psi$ uniform stability of trivial solutions for the nonlinear delayed system

$$
x^{\prime}(t)=f(t, x(t))+g(t, x(t-\tau(t)))
$$

and the nonlinear delayed Volterra integro-differential systems

$$
\begin{aligned}
x^{\prime}(t)= & f(t, x(t))+g(t, x(t-\tau(t))) \\
& +p(t, x(t)) \int_{0}^{t} q(s, x(s-\tau(s))) d s, \\
x^{\prime}(t)= & f(t, x(t))+g(t, x(t-\tau(t))) \\
& +p(t, x(t-\tau(t))) \int_{0}^{t} q(s, x(s)) d s,
\end{aligned}
$$

where $f, g, p, q \in C\left(\mathbb{R}_{+} \times \mathbb{R}^{n}, \mathbb{R}^{n}\right), f(t, 0)=g(t, 0)=$ $p(t, 0)=q(t, 0)=0$ for $t \in \mathbb{R}_{+}$, and $\tau \in C^{1}\left(\mathbb{R}_{+}, \mathbb{R}_{+}\right)$with 
$\tau(t) \leq t$ on $\mathbb{R}_{+}$. The systems studied in [7] do not include time delay, whereas all the systems studied in this paper have time delay.

In this paper, we investigate conditions on the functions $f, g, p, q$ under which the trivial solutions of systems (3), (4), and (5) are $\Psi$-stability on $\mathbb{R}_{+}$; the main tool used is the integral inequalities and the integral technique. Here $\Psi$ is a matrix function whose introduction allows us to obtain a mixed behavior for the components of solutions.

Let $\mathbb{R}^{n}$ denote the Euclidean $n$-space. For $x=\left(x_{1}, x_{2}\right.$, $\left.x_{3}, \ldots, x_{n}\right)^{T} \in \mathbb{R}^{n}$, let $\|x\|=\max \left\{\left|x_{1}\right|,\left|x_{2}\right|, \ldots,\left|x_{n}\right|\right\}$ be the norm of $x$. For an $n \times n$ matrix $A=\left(a_{i j}\right)$, we define the norm $|A|=\sup _{\|x\| \leq 1}\|A x\|$. It is well known that

$$
|A|=\max _{1 \leq i \leq n} \sum_{j=1}^{n}\left|a_{i j}\right|
$$

Let $\Psi_{i}: \mathbb{R}_{+} \rightarrow(0, \infty), \quad i=1,2, \ldots, n$, be continuous functions and $\Psi=\operatorname{diag}\left[\Psi_{1}, \Psi_{2}, \ldots, \Psi_{n}\right]$.

Now we give the definitions of $\Psi$-(uniform) stability that we will need in the sequel.

Definition 1 (see $[4,8]$ ). The trivial solution of (3) ((4) or (5)) is said to be $\Psi$-stable on $\mathbb{R}_{+}$if for every $\varepsilon>0$ and any $t_{0} \in \mathbb{R}_{+}$, there exists $\delta=\delta\left(\varepsilon, t_{0}\right)>0$ such that any solution $x(t)$ of (3) ((4) or (5)), which satisfies the inequality $\left\|\Psi\left(t_{0}\right) x\left(t_{0}\right)\right\|<\delta$, exists and satisfies the inequality $\|\Psi(t) x(t)\|<\varepsilon$ for all $t \geq t_{0}$.

Definition 2 (see $[4,8])$. The trivial solution of (3) ((4) or (5)) is said to be $\Psi$-uniformly stable on $\mathbb{R}_{+}$if it is $\Psi$-stable on $\mathbb{R}_{+}$ and the previous $\delta$ is independent of $t_{0}$.

\section{2. $\Psi$-Stability of the Systems}

To prove our theorems, we need the following lemmas.

Lemma 3. Let $h, k, p, q \in C\left(\mathbb{R}_{+} \times \mathbb{R}_{+}, \mathbb{R}_{+}\right)$with $(t, s) \mapsto$ $\partial_{t} h(t, s), \partial_{t} k(t, s), \partial_{t} p(t, s), \partial_{t} q(t, s) \in C\left(\mathbb{R}_{+} \times \mathbb{R}_{+}, \mathbb{R}_{+}\right)$. Assume, in addition, that $b \in C\left(\mathbb{R}_{+}, \mathbb{R}_{+}\right)$and $\alpha \in C^{1}\left(\mathbb{R}_{+}, \mathbb{R}_{+}\right)$ are nondecreasing functions and $\alpha(t) \leq t$ for $t \geq 0$. If $u \in$ $C\left(\mathbb{R}_{+}, \mathbb{R}_{+}\right)$satisfies

$$
\begin{aligned}
u(t) \leq & b(t)+\int_{0}^{t} h(t, s) u(s) d s+\int_{0}^{\alpha(t)} k(t, s) u(s) d s \\
& +\int_{0}^{t} p(t, s) u(s)\left(\int_{0}^{\alpha(s)} q(s, v) u(v) d v\right) d s
\end{aligned}
$$

for $t \geq 0$, and $b(t) \int_{0}^{t} R(s) Q(s) d s<1$, then

$$
u(t) \leq \frac{b(t) Q(t)}{1-b(t) \int_{0}^{t} R(s) Q(s) d s}, \quad t \geq 0
$$

where $Q(t)=\exp \left(\int_{0}^{t} h(t, s) d s+\int_{0}^{\alpha(t)} k(t, s) d s\right), R(t)=(d / d t)$ $\int_{0}^{t} p(t, s)\left(\int_{0}^{\alpha(s)} q(s, v) d v\right) d s$.
Proof. Let $T \geq 0$ be fixed and denote

$$
\begin{aligned}
x(t)= & \int_{0}^{t} h(t, s) u(s) d s+\int_{0}^{\alpha(t)} k(t, s) u(s) d s \\
& +\int_{0}^{t} p(t, s) u(s)\left(\int_{0}^{\alpha(s)} q(s, v) u(v) d v\right) d s, \quad t \geq 0,
\end{aligned}
$$

then $u(t) \leq b(t)+x(t)$, and $x$ is nondecreasing on $\mathbb{R}_{+}$. For $t \in[0, T]$, by calculations we get the following:

$$
\begin{aligned}
x^{\prime}(t)= & {\left[h(t, t) u(t)+\int_{0}^{t} \partial_{t} h(t, s) u(s) d s\right] } \\
& +\left[k(t, \alpha(t)) u(\alpha(t)) \alpha^{\prime}(t)+\int_{0}^{\alpha(t)} \partial_{t} k(t, s) u(s) d s\right] \\
& +\left[p(t, t) u(t) \int_{0}^{\alpha(t)} q(t, v) u(v) d v\right. \\
& \left.+\int_{0}^{t} \partial_{t} p(t, s) u(s)\left(\int_{0}^{\alpha(s)} q(s, v) u(v) d v\right) d s\right] \\
\leq & {[b(T)+x(t)]\left[\frac{d}{d t}\left(\int_{0}^{t} h(t, s) d s+\int_{0}^{\alpha(t)} k(t, s) d s\right)\right] } \\
& +[b(T)+x(t)]^{2} \frac{d}{d t} \int_{0}^{t} p(t, s)\left(\int_{0}^{\alpha(s)} q(s, v) d v\right) d s .
\end{aligned}
$$

Suppose that $b(0)>0$ (if $b(0)=0$, carry out the following arguments with $b(t)+\varepsilon$ instead of $b(t)$, where $\varepsilon>0$ is an arbitrary small constant, and subsequently pass to the limit as $\varepsilon \rightarrow 0$ to complete the proof), then we get

$$
\begin{aligned}
& \frac{x^{\prime}(t)}{[b(T)+x(t)]^{2}} \\
& \quad-\frac{1}{b(T)+x(t)} \frac{d}{d t}\left(\int_{0}^{t} h(t, s) d s+\int_{0}^{\alpha(t)} k(t, s) d s\right) \\
& \quad \leq \frac{d}{d t} \int_{0}^{t} p(t, s)\left(\int_{0}^{\alpha(s)} q(s, v) d v\right) d s .
\end{aligned}
$$

Let

$$
\begin{aligned}
z(t) & =\frac{1}{b(T)+x(t)}, \\
q(t) & =\int_{0}^{t} h(t, s) d s+\int_{0}^{\alpha(t)} k(t, s) d s, \\
Q(t) & =\exp (q(t)) \\
& =\exp \left(\int_{0}^{t} h(t, s) d s+\int_{0}^{\alpha(t)} k(t, s) d s\right), \\
R(t) & =\frac{d}{d t} \int_{0}^{t} p(t, s)\left(\int_{0}^{\alpha(s)} q(s, v) d v\right) d s,
\end{aligned}
$$


then, we have

$$
z^{\prime}(t)+z(t)\left(\frac{d}{d t} q(t)\right) \geq-R(t)
$$

Multiplying the above inequality by $e^{q(t)}=Q(t)$, we get

$$
\frac{d}{d t}(z(t) Q(t)) \geq-Q(t) R(t) .
$$

Consider now the integral on the interval $[0, t]$ to obtain

$$
z(t) Q(t) \geq z(0)-\int_{0}^{t} Q(s) R(s) d s, \quad 0 \leq t \leq T,
$$

so

$$
\begin{aligned}
z(t) & =\frac{1}{b(T)+x(t)} \\
& \geq\left[\frac{1}{b(T)}-\int_{0}^{t} Q(s) R(s) d s\right] \frac{1}{Q(t)} \\
& =\frac{1-b(T) \int_{0}^{t} Q(s) R(s) d s}{b(T) Q(t)}
\end{aligned}
$$

for $0 \leq t \leq T$. Let $t=T$, since $b(T) \int_{0}^{T} Q(s) R(s) d s<1$, then we have

$$
b(T)+x(T) \leq \frac{b(T) Q(T)}{1-b(T) \int_{0}^{T} Q(s) R(s) d s} .
$$

Since $T \geq 0$ was arbitrarily chosen, considering $u(t) \leq b(t)+$ $x(t)$, we get (8).

Lemma 4. Let $h, k, p, q, b, \alpha$ be as in Lemma 3. If $u \in$ $C\left(\mathbb{R}_{+}, \mathbb{R}_{+}\right)$satisfies

$$
\begin{aligned}
u(t) \leq & b(t)+\int_{0}^{t} h(t, s) u(s) d s+\int_{0}^{\alpha(t)} k(t, s) u(s) d s \\
& +\int_{0}^{\alpha(t)} p(t, s) u(s)\left(\int_{0}^{s} q(s, v) u(v) d v\right) d s
\end{aligned}
$$

for $t \geq 0$, and $b(t) \int_{0}^{t} R(s) Q(s) d s<1$, then

$$
u(t) \leq \frac{b(t) Q(t)}{1-b(t) \int_{0}^{t} R(s) Q(s) d s}, \quad t \geq 0
$$

where $Q(t)=\exp \left(\int_{0}^{t} h(t, s) d s+\int_{0}^{\alpha(t)} k(t, s) d s\right), R(t)=$ $(d / d t) \int_{0}^{\alpha(t)} p(t, s)\left(\int_{0}^{s} q(s, v) d v\right) d s$.

The proof is similar to the proof of Lemma 3, we omit the details.

Theorem 5. If there exist functions $a(t, s), b(t, s) \in C\left(\mathbb{R}_{+} \times\right.$ $\left.\mathbb{R}_{+}, \mathbb{R}_{+}\right)$with $(t, s) \mapsto \partial_{t} a(t, s), \partial_{t} b(t, s) \in C\left(\mathbb{R}_{+} \times \mathbb{R}_{+}, \mathbb{R}_{+}\right)$ such that

$$
\begin{gathered}
\|\Psi(t) f(s, x)\| \leq a(t, s)\|\Psi(s) x\|, \\
\|\Psi(t) g(s, x)\| \leq b(t, s)\|\Psi(s) x\|,
\end{gathered}
$$

for $0 \leq s \leq t$ and for all $x \in \mathbb{R}^{n}$. Moreover,

$$
\begin{gathered}
\limsup _{t \rightarrow \infty} \int_{0}^{t}(a(t, s)+b(t, s)) d s=L_{1}, \\
\left|\Psi(t) \Psi^{-1}(s)\right| \leq L_{2} \quad \text { for } 0 \leq s \leq t,
\end{gathered}
$$

and $|\Psi(t) x(\alpha(t))| \leq|\Psi(\alpha(t)) x(\alpha(t))|$, where $L_{1}, L_{2}$ are nonnegative constants. If $\alpha(t)=t-\tau(t)$ is an increasing diffeomorphism of $\mathbb{R}_{+}$. Then, the trivial solution of system (3) is $\Psi$-uniformly stable on $\mathbb{R}_{+}$.

Proof. Suppose that $x\left(t, t_{0}, x_{0}\right):=x(t)$ is the unique solution of system (3) which satisfies $x\left(t_{0}\right)=x_{0}$, since

$$
\begin{aligned}
x(t) & =x_{0}+\int_{t_{0}}^{t} f(s, x(s)) d s+\int_{t_{0}}^{t} g(s, x(\alpha(s))) d s \\
& =x_{0}+\int_{t_{0}}^{t} f(s, x(s)) d s+\int_{\alpha\left(t_{0}\right)}^{\alpha(t)} \frac{g\left(\alpha^{-1}(r), x(r)\right)}{\alpha^{\prime}\left(\alpha^{-1}(r)\right)} d r,
\end{aligned}
$$

after performing the change of variables $r=\alpha(s)$ in the second integral, and $\alpha^{-1}$ is the inverse of the diffeomorphism $\alpha$ then, it follows that

$$
\begin{aligned}
\|\Psi(t) x(t)\| \leq & \left\|\Psi(t) \Psi^{-1}\left(t_{0}\right) \Psi\left(t_{0}\right) x_{0}\right\| \\
& +\int_{t_{0}}^{t}\|\Psi(t) f(s, x(s))\| d s \\
& +\int_{\alpha\left(t_{0}\right)}^{\alpha(t)}\left\|\Psi(t) \frac{g\left(\alpha^{-1}(r), x(r)\right)}{\alpha^{\prime}\left(\alpha^{-1}(r)\right)}\right\| d s \\
\leq & L_{2}\left\|\Psi\left(t_{0}\right) x_{0}\right\|+\int_{t_{0}}^{t} a(t, s)\|\Psi(s) x(s)\| d s \\
& +\int_{\alpha\left(t_{0}\right)}^{\alpha(t)} \frac{b\left(t, \alpha^{-1}(r)\right)}{\alpha^{\prime}\left(\alpha^{-1}(r)\right)}\|\Psi(r) x(r)\| d r,
\end{aligned}
$$

this implies by Lemma 3 that

$$
\begin{aligned}
\|\Psi(t) x(t)\| \leq & L_{2}\left\|\Psi\left(t_{0}\right) x_{0}\right\| \exp \\
& \times\left(\int_{t_{0}}^{t} a(t, s) d s+\int_{\alpha\left(t_{0}\right)}^{\alpha(t)} \frac{b\left(t, \alpha^{-1}(r)\right)}{\alpha^{\prime}\left(\alpha^{-1}(r)\right)} d r\right) \\
= & L_{2}\left\|\Psi\left(t_{0}\right) x_{0}\right\| \exp \left(\int_{t_{0}}^{t}(a(t, s)+b(t, s)) d s\right) \\
\leq & L_{2} e^{L_{1}}\left\|\Psi\left(t_{0}\right) x_{0}\right\|,
\end{aligned}
$$

so for every $\varepsilon>0$, choose $\delta=\varepsilon /\left(L_{2} e^{L_{1}}\right)$, then

$$
\|\Psi(t) x(t)\| \leq L_{2} e^{L_{1}}\left\|\Psi\left(t_{0}\right) x_{0}\right\|<\varepsilon
$$

for $\left\|\Psi\left(t_{0}\right) x_{0}\right\|<\delta$ and for all $0 \leq t_{0} \leq t<\infty$. Hence, the conclusion of the theorem follows. 
Theorem 6. Let all the conditions in Theorem 5 hold. Suppose further that there exist functions $m(t, s), n(t, s) \in C\left(\mathbb{R}_{+} \times\right.$ $\left.\mathbb{R}_{+}, \mathbb{R}_{+}\right)$with $(t, s) \mapsto \partial_{t} m(t, s), \partial_{t} n(t, s) \in C\left(\mathbb{R}_{+} \times \mathbb{R}_{+}, \mathbb{R}_{+}\right)$ such that

$$
\begin{gathered}
\left\|\Psi(t) p(s, x) \Psi^{-1}(s)\right\| \leq m(t, s)\|\Psi(s) x\|, \\
\|\Psi(t) q(s, x)\| \leq n(t, s)\|\Psi(s) x\|,
\end{gathered}
$$

for $0 \leq s \leq t$ and for all $x \in \mathbb{R}^{n}$, moreover,

$$
\limsup _{t \rightarrow \infty} \int_{0}^{t} m(t, s)\left(\int_{0}^{s} n(s, u) d u\right) d s=L_{3}
$$

where $L_{3}$ is a nonnegative constant. Then, the trivial solutions of systems (4) and (5) are $\Psi$-uniformly stable on $\mathbb{R}_{+}$.

Proof. For that system (4), suppose $x\left(t, t_{0}, x_{0}\right):=x(t)$ is the unique solution of system (4) which satisfies $x\left(t_{0}\right)=x_{0}$, since

$$
\begin{aligned}
x(t)= & x_{0}+\int_{t_{0}}^{t} f(s, x(s)) d s+\int_{t_{0}}^{t} g(s, x(\alpha(s))) d s \\
& +\int_{t_{0}}^{t} p(s, x(s)) \int_{0}^{s} q(u, x(\alpha(u))) d u d s, \quad 0 \leq t_{0} \leq t,
\end{aligned}
$$

it follows that

$$
\begin{aligned}
\|\Psi(t) x(t)\| \leq & \left\|\Psi(t) \Psi^{-1}\left(t_{0}\right) \Psi\left(t_{0}\right) x_{0}\right\| \\
& +\int_{t_{0}}^{t}\|\Psi(t) f(s, x(s))\| d s \\
& +\int_{\alpha\left(t_{0}\right)}^{\alpha(t)} \frac{\left\|\Psi(t) g\left(\alpha^{-1}(r), x(r)\right)\right\|}{\alpha^{\prime}\left(\alpha^{-1}(r)\right)} d r \\
& +\int_{t_{0}}^{t}\left\|\Psi(t) p(s, x(s)) \Psi^{-1}(s)\right\| \\
& \times\left(\int_{0}^{\alpha(s)} \frac{\left\|\Psi(s) q\left(\alpha^{-1}(r), x(r)\right)\right\|}{\alpha^{\prime}\left(\alpha^{-1}(r)\right)} d r\right) d s \\
\leq & L_{2}\left\|^{t}\left(t_{0}\right) x_{0}\right\|+\int_{t_{0}}^{t} a(t, s)\|\Psi(s) x(s)\| d s \\
& +\int_{\alpha\left(t_{0}\right)}^{\alpha(t)} \frac{b\left(t, \alpha^{-1}(r)\right)}{\alpha^{\prime}\left(\alpha^{-1}(r)\right)}\|\Psi(r) x(r)\| d r \\
& +\int_{t_{0}}^{t} m(t, s)\|\Psi(s) x(s)\| \\
& \left.+\int_{0}^{\alpha(s)} \frac{n\left(s, \alpha^{-1}(r)\right)\|\Psi(r) x(r)\|}{\alpha^{\prime}\left(\alpha^{-1}(r)\right)} d r\right) d s
\end{aligned}
$$

after performing the change of variables $r=\alpha(s)$ (or $r=$ $\alpha(u))$ at some intermediate step, and $\alpha^{-1}$ is the inverse of the diffeomorphism $\alpha$. Denote

$$
\begin{aligned}
Q(t) & =\exp \left(\int_{t_{0}}^{t} a(t, s) d s+\int_{\alpha\left(t_{0}\right)}^{\alpha(t)} \frac{b\left(t, \alpha^{-1}(r)\right)}{\alpha^{\prime}\left(\alpha^{-1}(r)\right)} d r\right) \\
& =\exp \left(\int_{t_{0}}^{t}(a(t, s)+b(t, s)) d s\right), \\
R(t) & =\frac{d}{d t}\left[\int_{t_{0}}^{t} m(t, s)\left(\int_{0}^{\alpha(s)} \frac{n\left(s, \alpha^{-1}(r)\right)}{\alpha^{\prime}\left(\alpha^{-1}(r)\right)} d r\right) d s\right] \\
& =\frac{d}{d t}\left[\int_{t_{0}}^{t} m(t, s)\left(\int_{0}^{s} n(s, u) d u\right) d s\right] .
\end{aligned}
$$

This implies by Lemma 3 that

$$
\begin{aligned}
& \|\Psi(t) x(t)\| \\
& \leq L_{2}\left\|\Psi\left(t_{0}\right) x_{0}\right\| \frac{Q(t)}{1-L_{2}\left\|\Psi\left(t_{0}\right) x_{0}\right\| \int_{0}^{t} Q(v) R(v) d v} \\
& \leq\left\|\Psi\left(t_{0}\right) x_{0}\right\| \frac{L_{2} e^{L_{1}}}{1-L_{2}\left\|\Psi\left(t_{0}\right) x_{0}\right\| e^{L_{1}} \int_{0}^{t} R(v) d v} \\
& =\left\|\Psi\left(t_{0}\right) x_{0}\right\| \\
& \quad \times \frac{1-L_{2}\left\|\Psi\left(t_{0}\right) x_{0}\right\| e^{L_{1} \int_{t_{0}}^{t} m(t, s)\left(\int_{0}^{s} n(s, u) d u\right) d s}}{\leq}\left\|\Psi\left(t_{0}\right) x_{0}\right\| \frac{L_{2} e^{L_{1}}}{1-L_{2} L_{3}\left\|\Psi\left(t_{0}\right) x_{0}\right\| e^{L_{1}}}
\end{aligned}
$$

for $L_{2} L_{3}\left\|\Psi\left(t_{0}\right) x_{0}\right\| e^{L_{1}}<1$ and $0 \leq t_{0} \leq t$. So, for every $\varepsilon>0$ and $t_{0} \geq 0$, let $0<q<1 / L_{2} L_{3} e^{L_{1}}$ be a constant and choose $\delta=\min \left\{q,\left(\left(1-q L_{2} L_{3} e^{L_{1}}\right) \varepsilon\right) / L_{2} e^{L_{1}}\right\}$, then

$$
\|\Psi(t) x(t)\|<\frac{\left(1-q L_{2} L_{3} e^{L_{1}}\right) \varepsilon}{L_{2} e^{L_{1}}} \times \frac{L_{2} e^{L_{1}}}{1-q L_{2} L_{3} e^{L_{1}}}=\varepsilon
$$

for $\left\|\Psi\left(t_{0}\right) x_{0}\right\|<\delta$ and for all $0 \leq t_{0} \leq t<\infty$. This proves that the trivial solution of system (4) is $\Psi$-uniformly stable on $\mathbb{R}_{+}$.

Using Lemma 4, the proof of system (5) is similar to that of system (4) and the details are left to the readers.

Remark 7. For $\Psi_{i}=1, i=1,2, \ldots, n$, we obtain the theorems of classical stability and uniform stability.

\section{Examples}

Example 8. Consider the nonlinear differential system

$$
\begin{aligned}
& x_{1}^{\prime}(t)=x_{1}(t)+x_{1}\left(\frac{t}{2}\right) \sin t, \\
& x_{2}^{\prime}(t)=-x_{2}(t)+x_{2}\left(\frac{t}{2}\right) \cos t .
\end{aligned}
$$


In (33), $f(t, x(t))=\left(x_{1}(t),-x_{2}(t)\right)^{T}, g(t, x(t / 2))=\left(x_{1}(t / 2)\right.$ $\left.\sin t, x_{2}(t / 2) \cos t\right)^{T}$. Let $\Psi(t)=\left(\begin{array}{cc}e^{-t} & 0 \\ 0 & e^{-t}\end{array}\right)$, then $a(t, s)=$ $b(t, s)=e^{-(t-s)}$ for $0 \leq s \leq t \leq \infty$, it is easy to verify that $L_{1}=2, L_{2}=1$, and all the assumptions in Theorem 5 satisfied, so the trivial solution of system (33) is $\psi$-uniformly stable on $\mathbb{R}_{+}$.

Example 9. Consider the nonlinear Volterra integro-differential system as follows:

$$
\begin{aligned}
& x_{1}^{\prime}(t)=x_{1}(t)+x_{1}(t) e^{-t} \int_{0}^{t} x_{1}\left(\frac{s}{2}\right) \cos s d s, \\
& x_{2}^{\prime}(t)=-x_{2}(t)+x_{2}(t) e^{-t} \int_{0}^{t} x_{2}\left(\frac{s}{2}\right) \sin s d s .
\end{aligned}
$$

In (34), $f(t, x(t))=\left(x_{1}(t),-x_{2}(t)\right)^{T}, g \equiv 0, p(t, x(t))=$ $\left(x_{1}(t) e^{-t}, x_{2}(t) e^{-t}\right)^{T}, q(s, x(s / 2))=\left(x_{1}(s / 2) \cos s, x_{2}(s / 2)\right.$ $\sin s)^{T}$. Choose the same matrix function $\Psi(t)$, then $a(t, s)=$ $n(t, s)=e^{-(t-s)}, b(t, s) \equiv 0, m(t, s)=e^{-2(t-s)}$ for $0 \leq s \leq t \leq$ $\infty$, it is easy to verify that $L_{1}=L_{2}=1, L_{3}=1 / 2$, and all the assumptions in Theorem 6 are satisfied, so the trivial solution of system (34) is $\psi$-uniformly stable on $\mathbb{R}_{+}$.

\section{Acknowledgments}

The authors are very grateful to the referees for their valuable comments and suggestions, which helped to shape the paper's original form. This research was supported by the NNSF of China (10971139), NSF of Shandong Province (ZR2012AL03) and the Shandong Education Fund for College Scientific Research (J11LA51).

\section{References}

[1] O. Akinyele, "On partial stability and boundedness of degree $k$," Atti della Accademia Nazionale dei Lincei. Rendiconti. Classe di Scienze Fisiche, Matematiche e Naturali, vol. 65, no. 6, pp. 259264, 1978.

[2] A. Constantin, Asymptotic Properties of Solutions of Differential Equations, vol. 3 of Seria Stiinte Matematice, Analele Universitãtii Din Timisoara, 1992.

[3] J. Morchało, “On $\left(\psi, L_{p}\right)$-stability of nonlinear systems of differential equations, Analele Stiintifice ale Universitàtii "AI. I. Cuza” Iasi, Tomul XXXVI, s. I-a,”, Matematicã, vol. 36, no. 4, pp. 353-360, 1990.

[4] A. Diamandescu, "On the $\Psi$-asymptotic stability of a nonlinear Volterra integro-differential system," Bulletin Mathématique de la Société des Sciences Mathématiques de Roumanie, vol. 46(94), no. 1-2, pp. 39-60, 2003.

[5] A. Diamandescu, "On the $\psi$-stability of a nonlinear Volterra integro-differential system," Electronic Journal of Differential Equations, vol. 2005, no. 56, pp. 1-14, 2005.

[6] F. M. Dannan and S. Elaydi, "Lipschitz stability of nonlinear systems of differential equations," Journal of Mathematical Analysis and Applications, vol. 113, no. 2, pp. 562-577, 1986.

[7] F. W. Meng, L. Z. Li, and Y. Z. Bai, " $\Psi$-stability of nonlinear Volterra integro-differential systems," Dynamic Systems and Applications, vol. 20, no. 4, pp. 563-574, 2011.
[8] V. Lakshmikantham and M. R. M. Rao, "Stability in variation for nonlinear integro-differential equations," Applicable Analysis, vol. 24 , no. 3, pp. 165-173, 1987. 


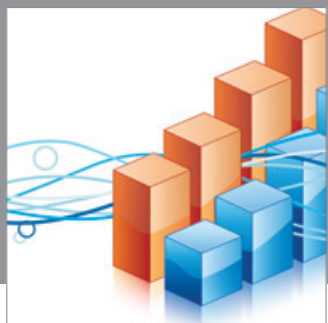

Advances in

Operations Research

mansans

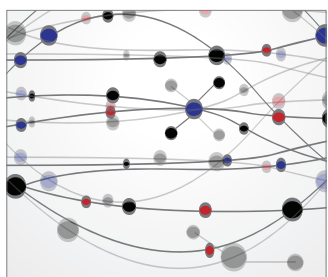

The Scientific World Journal
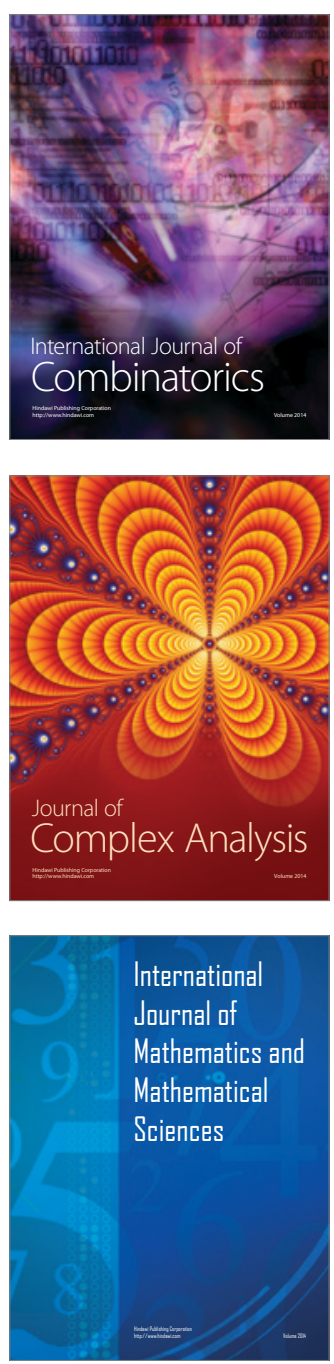
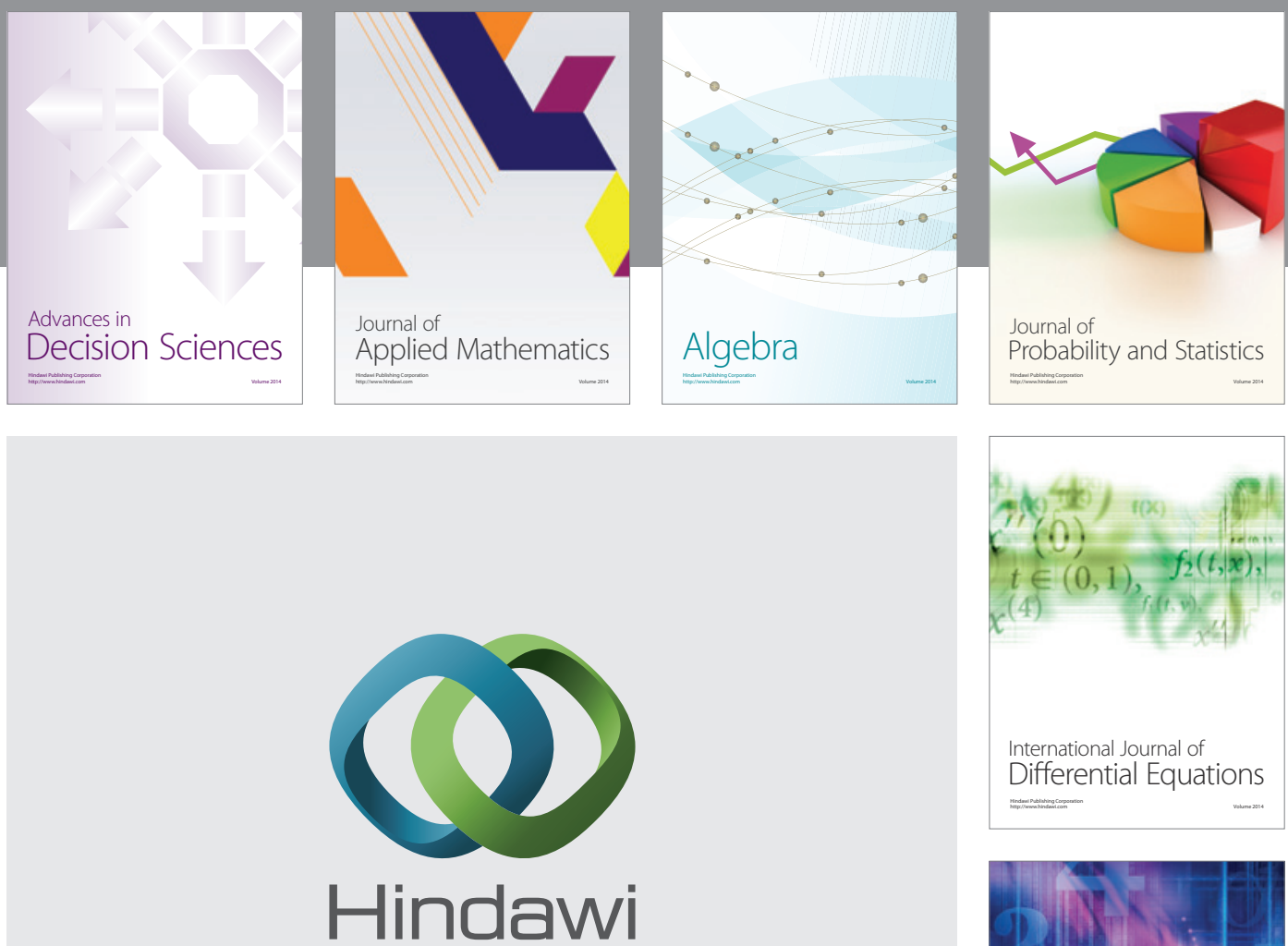

Submit your manuscripts at http://www.hindawi.com
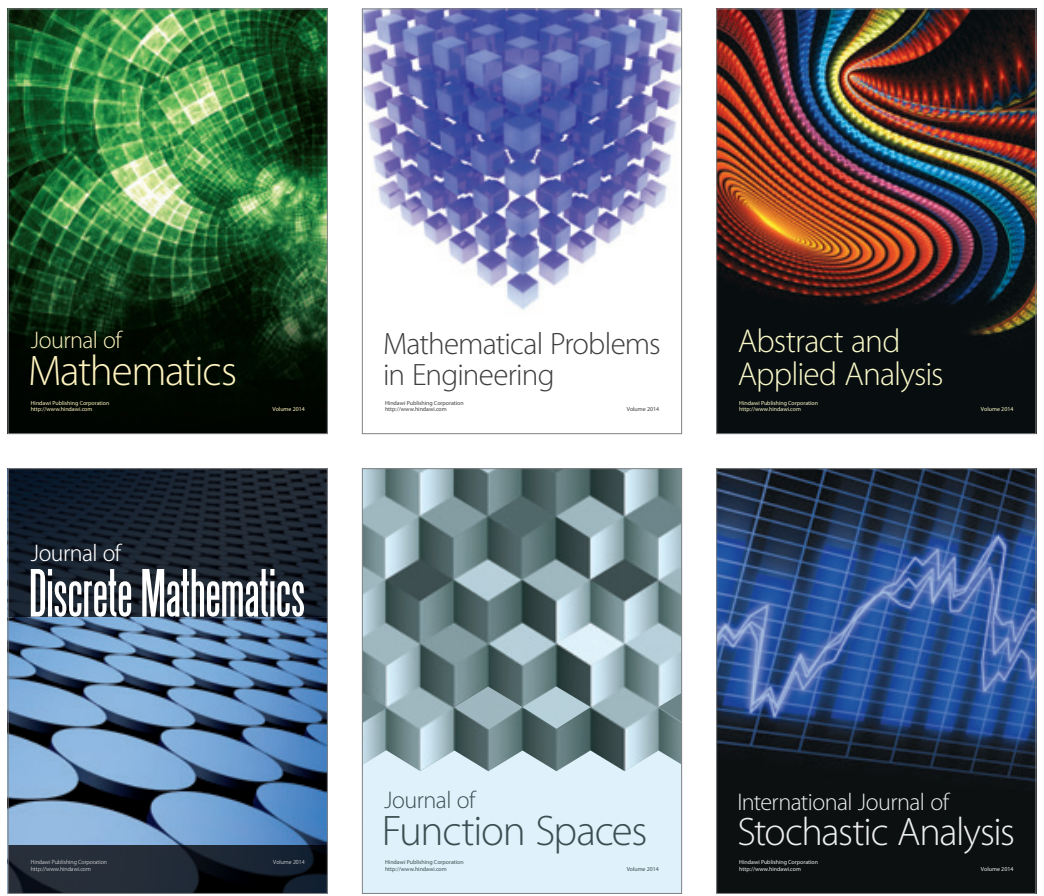

Journal of

Function Spaces

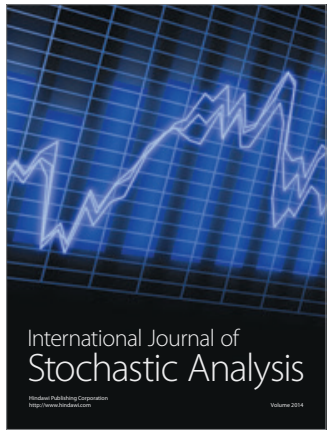

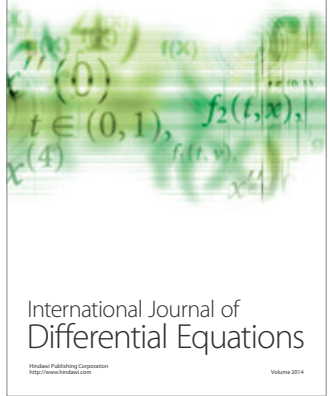
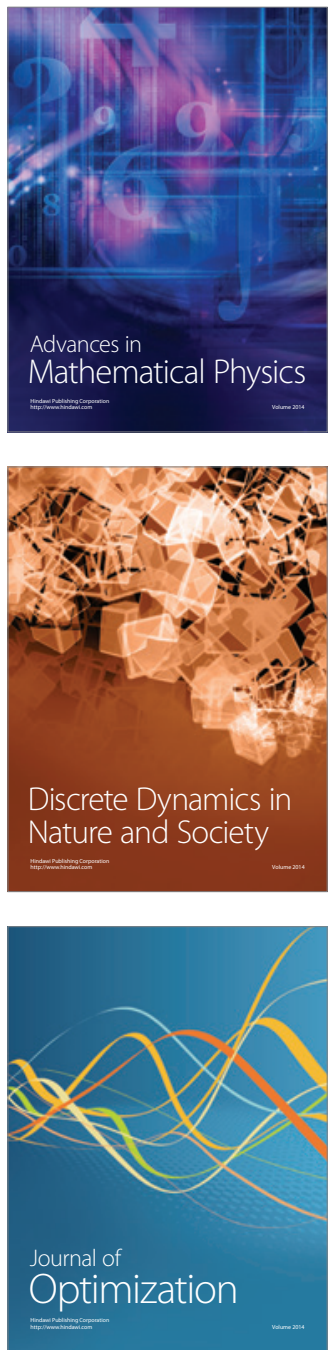